\title{
BATTAGE DE PIEUX TUBULAIRES EN MILIEU SABLEUX CAPACITE PORTANTE ET ROLE DE LA COLONNE DE SOL
}

\author{
B. DALI . B. HERITIER \\ CEBTP - Domaine Saint PQUI - BP 37 - 78470 SAINT REMY LES CHEVREUSE
}

\begin{abstract}
Despite the improvements of driveability prediction of piles, many difficulties are still encountered with the open ended tubular pile. In addition to the classical problems, the presense of a column of soil induces a complex phenomena. During the driving, the soil is either coupled to the tube either in friction.

This paper presents a case study of a full-scale test. The method SIMBAT developped by the C.E.B.T.P. is applied to determine the static bearing capacity of a tubular piles and the analysis allows the comprehension of the behaviour of the pile during the driving.
\end{abstract}

\section{INTRODUCTION}

En France. les pieux battus représentent une part limitée des pieux réalisés chaque année, par contre ils sont largement utilisés dans les projets portuaires et offshore. Un des handicaps reste sans doute la difficulté de prédire la capacité portante statique, en particulier à partir des résultats du battage. Les résistances calculées à partir des formules de battage sont en général très dispersées et les résultats aléatoires (Bustamante. 1985). On a même pu lire que la meilleure prévision de battage sur. un site donné était celle faite à partir des. abaques de dimensionnement des pleux chargés statiquement.

Les recherches conduites ces dernières années ont fait progresser les connaissances pour la détermination de la charge statique à partir des essais dynamiques (battage, post-battage, chargement dynamique).

Dans le cas des pieux en béton ou en métal fermés à la base. la prévision de la capacité statique est satisfaisante, par contre pour les pieux ouverts, le problème est beaucoup plus complexe en raison de la présence du bouchon:

- Les pieux fermés ont en général un profil et une impédance réguliers. En cas d'anomalies pour les pieux béton, il est nécessaire d'introduire l'impédance réelle, ce qui complique linterprétation, mais ce qui est possible à partir de la détermination du profil d'impédance (Paquet, 
- Avec les pieux ouverts la colonne de sol remplit une partie du pieu. Lorsqu'elle est solidaire, le comportement est bouchonnant, mais alors quelle est l'impédance du pieu ? Lorsqu'elle est libre, partiellement, puisqu'il y a frottement, le comportement est carottant, comment modéliser dans ce cas.

Plusieurs tentatives de modélisation ont été faites et les modèles s'améliorent mais ils ne seront fiables que lorsqu'ils auront été validés avec des cas variés, dans des conditions géotechniques différentes.

Cet article présente le cas d'un site ou plusieurs pleux tubulaires ont été battus et ou le type d'analyse fait à partir de la méthode SIMBAT du CEBTP permet d'identifier au cours du post-battage le comportement des pieux et de la colonne de sol et de proposer une capacité portante équivalente.

\section{RAPPEL SOMMAIRE DE LA METHODE SIMBAT}

SIMBAT est une méthode globale de chargement dynamique des pieux qui permet de déterminer la courbe charge-enfoncement statique équivalente et. à partir du critère de rupture choisi, la capacité portante d'un pieu.

Les principes généraux ont été présentés dans l'article de PAQUET (1988) et la méthode décrite dans un article suivant (HERITIER - 1991).

Un essai SIMBAT comprend les séquences suivantes: chargement dynamique du pieu et acquisition des données, validation des résultats, calcul de la résistance dynamique, détermination de la courbe charge-enfoncement.

\section{Chargement dynamique}

Le pieu est en place et l'essai est un post-battage pour un pieu battu, un chargement dynamique pour un pieu foré. Les chocs sont générés par une masse tombant en chute libre, ce qui présente de nombreux avantages: hauteur de chute variable, forme régulière du signal de force, séquence de chocs contrôlée.

Le battage est possible avec les marteaux utilisés pour le chantier mais ils niont pas la même souplesse d'utilisation.

La tête du pieu, qui sent de dynamomètre, est instrumentée (jauges. accéléromètres, théodolite électroniaue) - fiaure 1-. 
Les données sont acquises et traitée en temps réel.

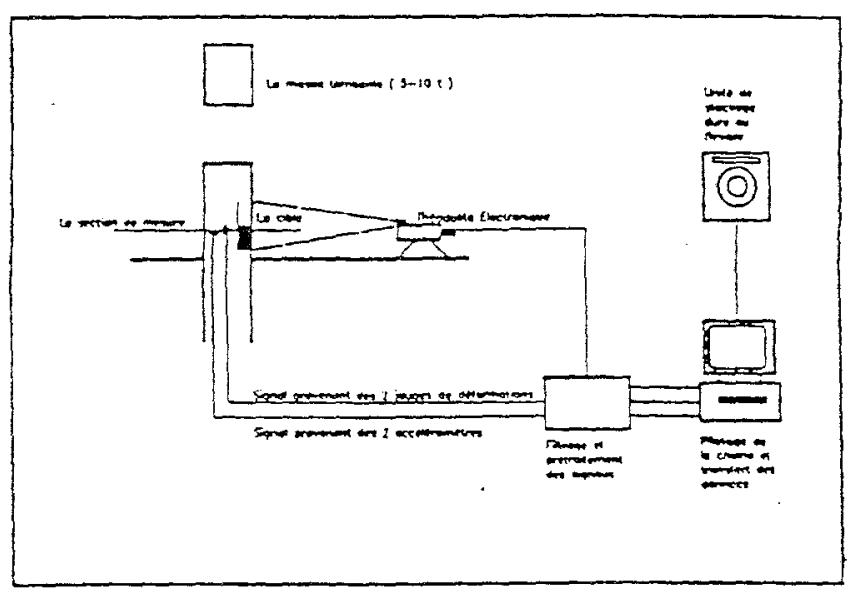

Fig. 1 : Principe d'acquisition de l'essai SIMBAT

\section{Validation des signaux}

Cette étape est essentielle pour l'interprétation des essais. Au cours d'une séquence de battage, dans un horizon géotechnique donné, un régime d'équilibre s'établit. II correspond à une résistance dynamique du sol qui varie en fonction de la profonceur et de la vitesse de pénétration du pieu. Le pieu étant au repos, le sol se cicatrise et son état final dépend du temps de repos et du type de sol.

L'analyse d'une centaine de cas montre qu'un post-battage implique de retrouver un état d'équilibre à un niveau déterminé. Un choc isolé ne peut pas rendre compte correctement de la résistance dynamique maximale.

\section{Calcui de la résistance dynamique}

Le calcul direct de la résistance dynamique Ray est fait à partir de la décomposition des signaux mesurés, ce qui est identique mais plus direct que la formule de CASE. La figure 2 donne un exemple de décomposition. 
Le calcul final est fait par modélisation.

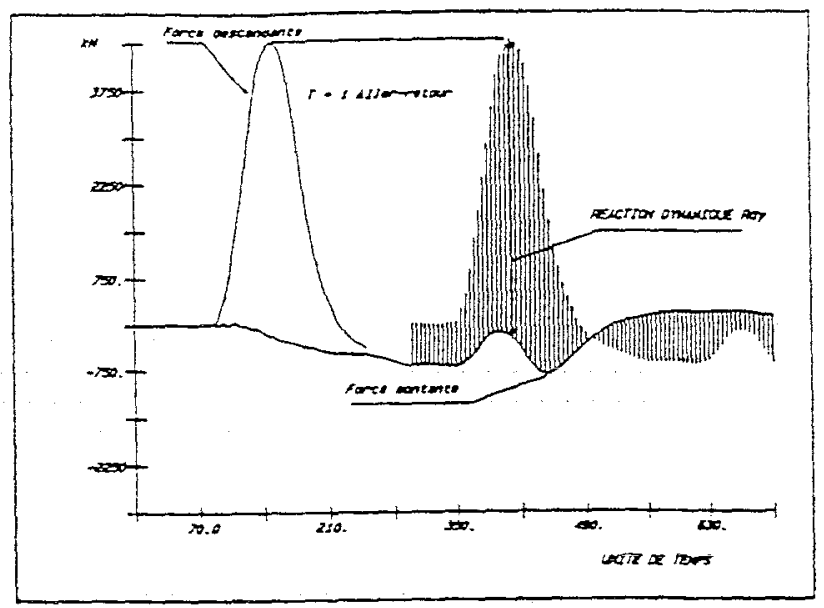

Fig.2

\section{Courbe charge-enfoncement}

A partir d'une séquence de choc et du calcul des paramètres Ray et $e_{p}$. enfoncement permanent pour un choc, it est possible de déuire la résistance statique équivalente, Rst qui correspond à un enfondement permanent nul.

La relation proposée par PAQUET (1988) a été complétée à partir de l'étude de plusieurs cas de chargement et peut s'écrire sous la forme:

$$
R_{d y}=R_{s t}+f\left(e_{p}\right)=R_{s t}+\left(K . e_{p}\right)^{\alpha}
$$

avec :

$$
\begin{aligned}
& e_{p}=\text { enfoncement permanent } \\
& K=\text { paramètre du pieu } \\
& \alpha=\text { paramètre fonction du sol } .
\end{aligned}
$$

Ces deux paramètres sont déterminés expérimentalement

\section{DIFFICULTES RENCONTREES AVEC LES TUBES OUVERTS POUR LA MODELISATION DE LA CAPACITE PORTANTE STATIQUE EQUIVALENTE}

Ces essais de chargement dynamique ont été effectués sur des pieux dans l'argile des flandres. Les pieux d'une longueur de $12 \mathrm{~m}$ avaient été battus ou forés dans un terrain constitué par $2 \mathrm{~m}$ de limon de couverture surmontant l'argile des flandres. Le tableau 1 ci-dessous donne pour chaque pleu la capacité partante de l'essai statique et les valeurs équivalentes calculées à partir des essais de chargement dynamique pendant la phase de développement de la méthode SIMBAT. 


\begin{tabular}{|c|c|c|c|}
\hline Type & Dimensions & $\begin{array}{c}\text { Capacité } \\
\text { portante statique } \\
-k N\end{array}$ & $\begin{array}{c}\text { Capacité équivalente par } \\
\text { essais dynamiques - } k N\end{array}$ \\
\hline $\begin{array}{c}\text { Béton tarière } \\
\text { creuse }\end{array}$ & $\varnothing=0,50 \mathrm{~m}$ & 1300 & 1400 \\
$\begin{array}{c}\text { Caisson } \\
\text { fermé } \\
\text { Tube ouvert }\end{array}$ & $\varnothing, 53 \times 0,39$ & 1300 & $1200-1500$ \\
\hline
\end{tabular}

Tableau 1 : Prévision dans l'argile des Flandres

II faut noter que les essais de chargement dynamiques sont postérieurs de 6 mois aux essais statiques.

La concordance est satisfaisante en ce qui concerne les pieux bétons ou fermé à la base avec un léger accroissement de la capacité portante au cours du temps. Par contre la prévision est incorrecte avec le tube ouvert. Les difficultés de modélisation proviennent essentiellement de la présence du bouchon de sol. On peut également observer que dans une argile surconsolidée, comme largile des flandres, la "cicatrisation" du milieu peut jouer un rôle important. Les prévisions faites sur un autre site, avec des pieux forés, dans la même formation géotechnique, ont donné des résultats très satisfaisants.

\section{ESSAIS DE PIEUX EN MILIEU SABLEUX COMPORTEMENT DE LA COLONNE DE SOL}

\section{Concitions expérimentales}

Les pieux ont été battus dans un sable fin saturé de densité moyenne. Ils sont du type métallique tubulaire ouvert avec les caractéristiques suivantes:

diamètre : $\varnothing=0.60 \mathrm{~m}$

épaisseur: $e=9.52 \mathrm{~mm}$

longueur: $1=8.30 \mathrm{~m}$ avec $7,30 \mathrm{~m}$ de fiche.

Le battage a été fait avec un marteau diesel.

Le post-battage a été fait selon la méthodologie SIMBAT avec une masse tombante de $8 \mathrm{t}$. Une tête spéciale a été confectionnée pour coiffer la tête du pieu avec un coussin en bois pour le battage. 


\section{Résultats des essais}

La tête du pieu a été soumise à une série de chocs.

La figure 3 donne un exemple de courbes enregistrées et calculées pour un choc donné :
A : accélération
B : vitesse de la tête
C : enfoncement de la tête
D : force d'impact

$\hat{A}$

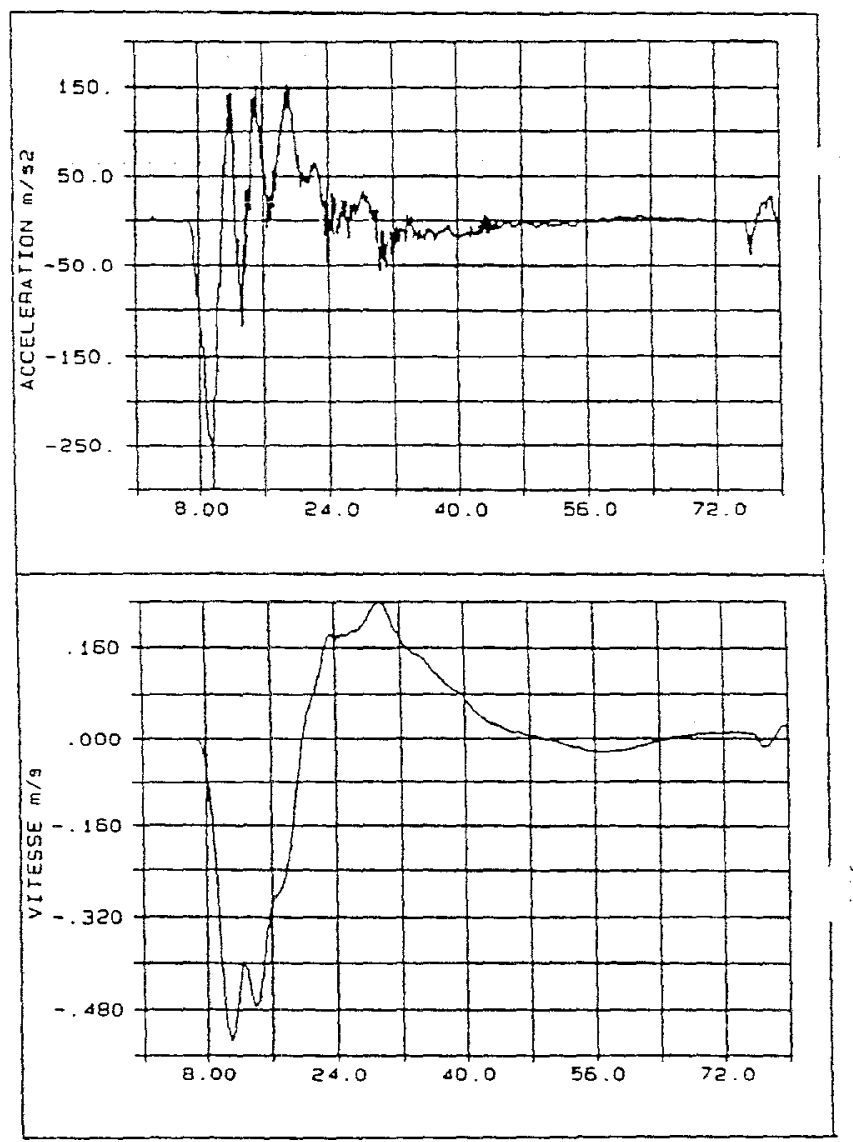


C
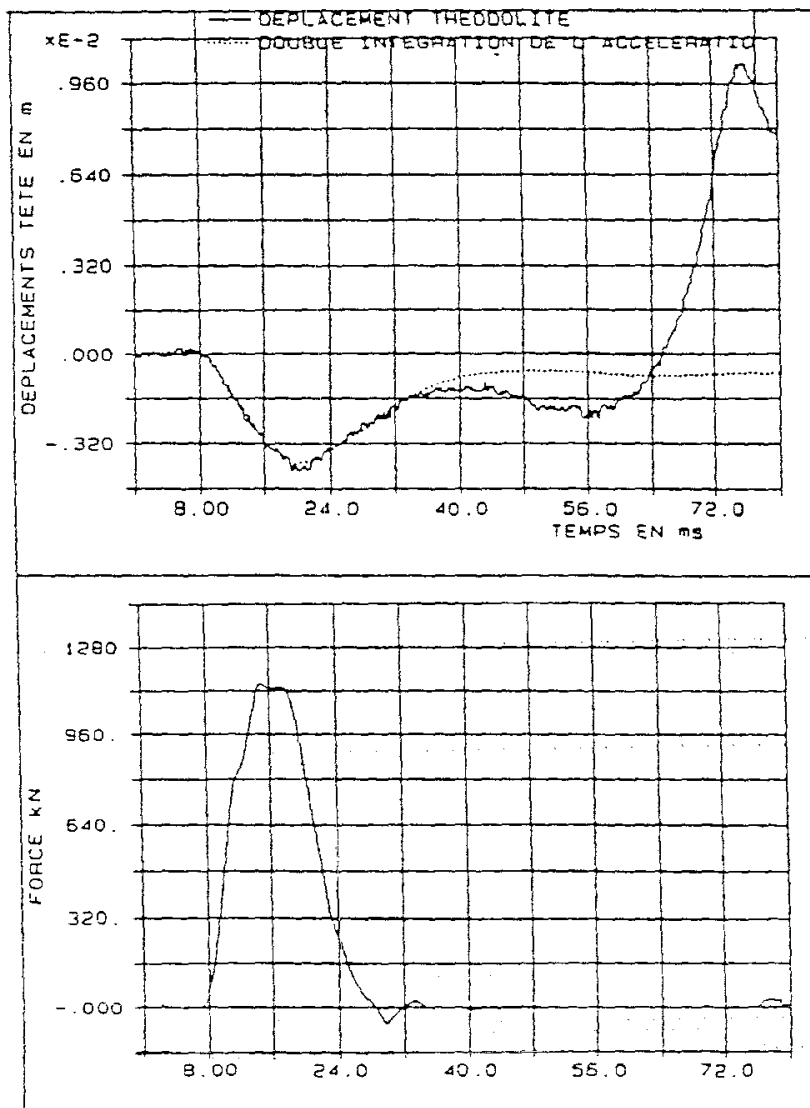

D

Fig. 3 
On observe pour le théodolite le problème de basculement au passage des ondes de Rayleigh, ce qui rend le signal du déplacement inexploitable audelà de 56 ms dans ce cas, mais la partie utile est largement suffisante pour linterprétation des mesures.

\begin{tabular}{|c|c|c|c|}
\hline No du choc & Hauteur $(\mathrm{m})$ & $E p(\mathrm{~mm})$ & Rdy $(\mathrm{kN})$ \\
\hline 1 & 0,3 & 0,70 & 1,152 \\
2 & 0,2 & 0,08 & 0,868 \\
3 & 0,3 & 0,40 & 1,247 \\
4 & 0,5 & 1,00 & 1,583 \\
5 & 0,8 & 3,72 & 1,883 \\
6 & 0,5 & 0,08 & 1,574 \\
7 & 0,8 & 4.28 & 1,810 \\
8 & 0,5 & 2,40 & 1,550 \\
9 & 0.7 & 1,64 & 1,500 \\
10 & 0.5 & 0,84 & 1,297 \\
11 & 0.7 & 2,56 & 1,710 \\
12 & 0,4 & 1,08 & 1,420 \\
13 & 0,5 & 2,00 & 1,550 \\
14 & 0,3 & 0,40 & 1,390 \\
15 & 0,5 & 2,84 & 1,744 \\
16 & 0,7 & 2,56 & 1,852 \\
17 & 0,4 & 2,40 & 1,760 \\
18 & 0,5 & 2,00 & 1,720 \\
19 & 0,4 & 1,48 & 1,770 \\
20 & 0,5 & 2,44 & 1,940 \\
\hline
\end{tabular}

Tableau 2

Les résultats du post-battage sont récapitulés dans le tableau 2 qui donne pour chaque choc le déplacement permanent et la réaction dynamique calculée à partir de la décomposition des signaux.

\section{Analyse directe des résultats}

Ce type d'analyse est fait en cours d'essai et permet une analyse directe de la séquence de post-battage.

A partir de la séquence de chocs (tableau 2), la construction du diagramme $R_{d y}-e_{p}$, figure 4 , met en évidence deux régimes distincts après les chocs de mise en place ( 1 à 3 ):

- Le premier correspond à la série de chocs 4 à 7 avec une résistance dynamique Rdy pour un enfoncement nul voisine de $1500 \mathrm{kN}$.

- Le secona, qui correspond à la série de chocs 8 à 15 donne une valeur de Ray plus faible voisine de $1300 \mathrm{KN}$. 
- On retrouve à partir du $16 e$ choc une résistance plus forte. de l'ordre de $1600 \mathrm{KN}$ qui semble saccroitre avec la profondeur.

Des régimes encore plus différenciés avaient été obtenus au cours d'une expérience antérieure sur un site voisin.

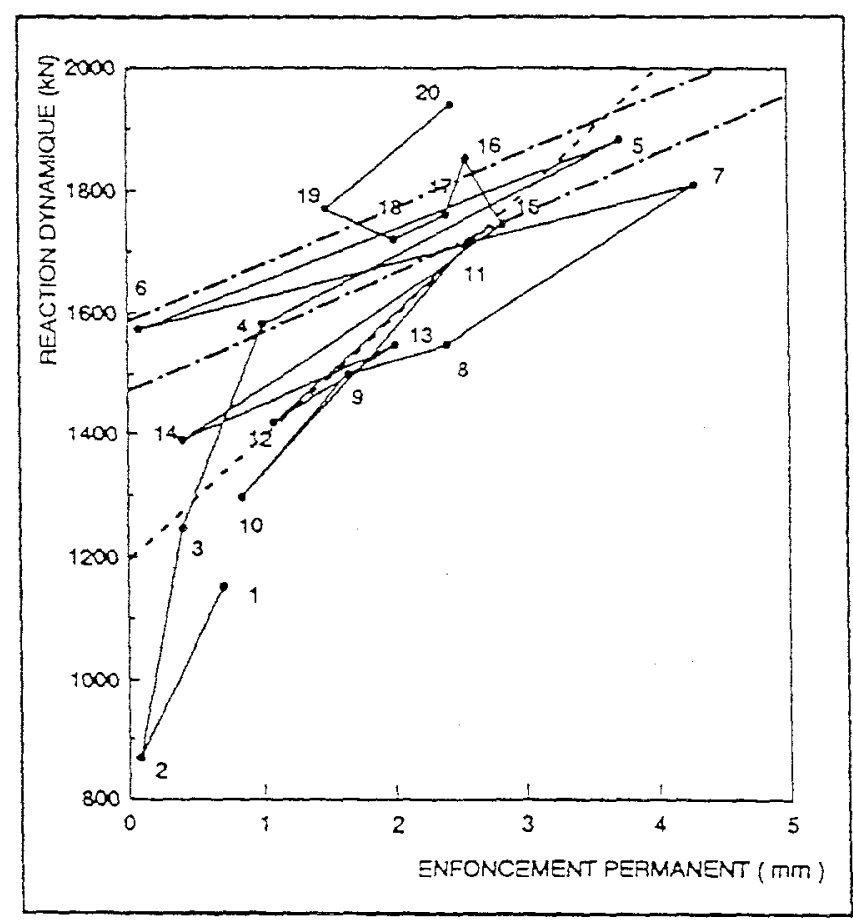

Fig.4

\section{Analyse por modélisation}

Paquet (1988) a montré que le calcul direct de la résistance dynamique par la formule de case ou par décomposition des signaux donnait une approximation de la véritable résistance dynamique du sol, approximation en générale suffisante sur chantier.

L'analyse directe est complétée par un programme de calcul où le comportement du pleu est modélisé à partir des signaux enregistrés. le calage étant fait sur le déplacement permanent du pieu, mesuré qu théodolite, le choix de la vitesse du pieu pour le calage pouvant conduire à des résultats erronés.

La modélisation ne tient pas compte de la colonne de sol mais elle permet de simuler les mécanismes en jeu:

- le comportement bouchonnant est simulé par une forte réaction en pointe et un frottement latéral faible

- le comportement carottant par un effort en pointe réduit et une distribution plus forte du frottement le long du pieu 
Ces deux modèles sont représentés sur la figure 5 .

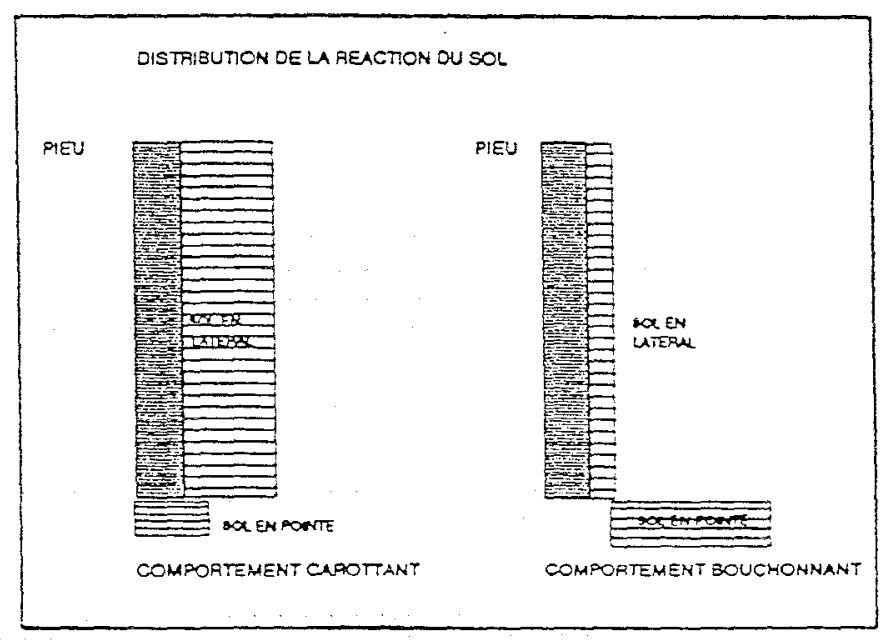

Fig. 5

Un exemple de modélisation est donné sur la figure 6 . On observe la bonne concordance avec les signaux de vitesse (fig.6A) et de déplacement (fig. $6 \mathrm{~B}$ ).

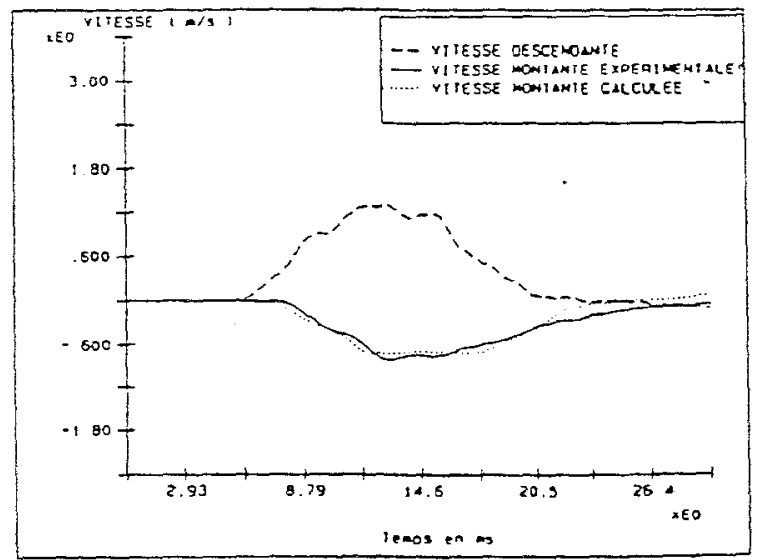

Fig. $6 \mathrm{~A}$ 


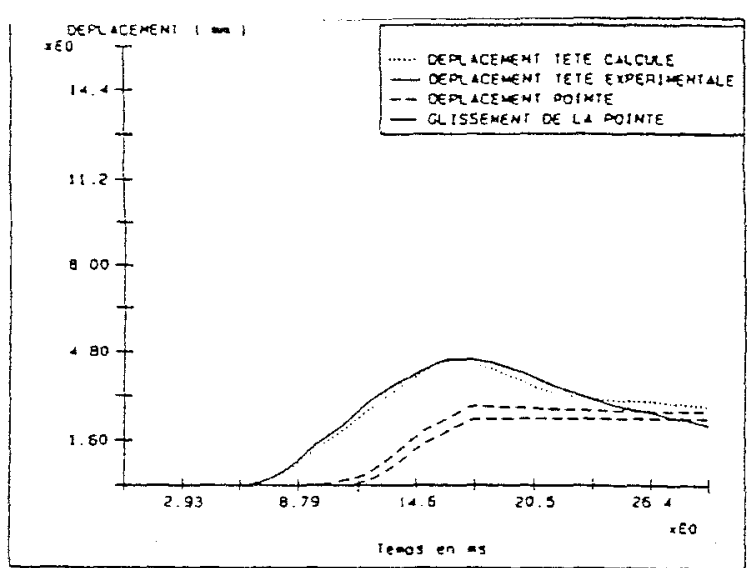

Fig.6B

Les résultats d'ensemble permettent d'établir le tableau suivant:

\begin{tabular}{|c|c|}
\hline Chocs no & Comportement \\
\hline $1 a b$ & bouchonnant \\
$7 a 13$ & carottant \\
$14 a 20$ & bouchonnant \\
\hline
\end{tabular}

et la figure 7 qui donne la courbe charge statique équivalente-enfoncement met bien en évidence les différents régimes au cours du battage.

La capacité portante proposée pour ce pieu est de $1500 \mathrm{KN}$, si l'on avait suivi les recommandations issues du projet de recherche CLAROM (Le Tirant1991), qui propose pour la charge statique équivalente en milieu sableux de prendre les valeurs où le pieu est bouchonnant, la valeur retenue aurait été 1600 KN.

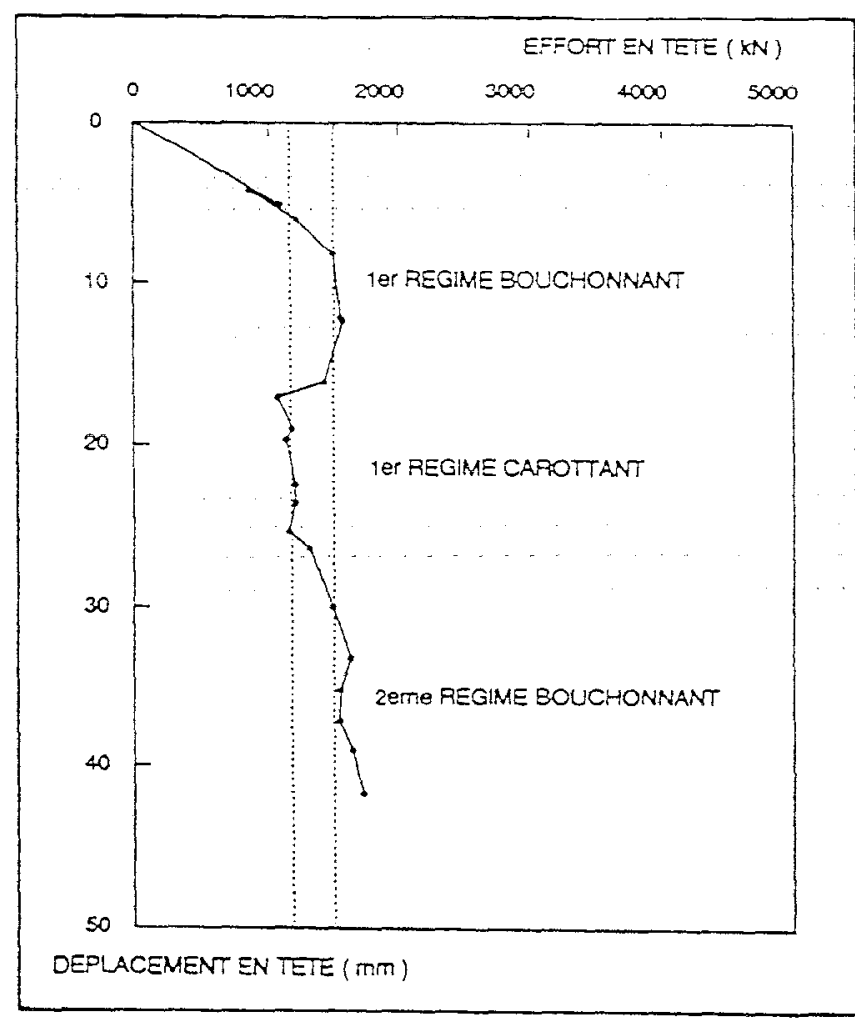

Fig.7 


\section{CONCLUSIONS}

Les essais de chargement dynamique de pieux tubulaires réalisés en milieu sableux ont permis d'améliorer la compréhension du phénomène de bouchon en cours de battage:

- l'instrumentation d'un pieu, en dehors de la vérification de l'efficacité du dispositif de battage est un moyen efficace de mesurer la résistance dynamique du sol

- à partir de l'analyse d'une séquence de post-battage, il est également possible de déterminer la capacité statique équivalente du pieu.

Dans le cas présent, deux comportements ont été mis en évidence. Ils correspondent à la formation du bouchon avec un comportement carottant ou bouchonnant et aux situations intermédiaires.

Pour améliorer la compréhension du phénomène, des essais sur des sites variés sont nécessaires.

Leur interprétation implique une instrumentation de qualité et une méthode d'essai rigoureuse.

\section{REFERENCES BIBLIOGRAPHIQUES}

(1) BUSTAMANTE M., FRANK R., GIANESELI L., KLOS J. "Vérification expérimentale des formules de battage", Xle Congrès Int. Méca.Sols. Tr. Fond, San Francisco, août 1985.

(2) PAQUET Jean, "Une nouvelle orientation dans le contrôle d'intégrité des pieux par sollicitation dynamique : le profil d'impédance". Colloque "Fondations Profondes", ENPC. Paris, 19-21 mars 1991.

(3) PAQUET Jean. "Checking Bearing Capacity by Dynamic Loading - Choice of a methodology", 3rd, Conference on the Application of Stress Wave Theory on Piles - Ottawa - Canada-mai 1988.

(4) HERITIER B., PAQUET J., DALI B., "SIMBAT: Une méthode rapide d'évaluation de la capacité portante des pieux.". Colloque "Fondations Profondes", ENPC, PARIS, 19-21 MARS 1991. 
(5) LE TIRANT P., NAUROY J-F., BRUCY F., "Dimensionnement des pieux de fondation offshore dans les sables denses", Colloque "Fondations Profondes" . ENPC. Paris, 19-21 mars 1991.

(6) BRUCY F., NAUROY J-F., LE TIRANT P., MEUNIER J., "Comparaison d'essais dynamiques et statiques de pieux dans la sables", Colloque "Fondations Profondes", ENPC, Paris, 19-21 mars 1991.

(7) MEUNIER J., BRUCY F., PAQUET J., "Apport de linstrumentation en cours de battage. Application à quatre pieux expérimentaux dans le sable", Colloque "Fondations Profondes", ENPC Paris, 19-21 mars 1991. 